\title{
Intrastromal fluid drainage with air tamponade: anterior segment optical coherence tomography guided technique for the management of acute corneal hydrops
}

\author{
Rasik B Vajpayee, ${ }^{1,2}$ Prafulla K Maharana, ${ }^{1}$ Luci Kaweri, ${ }^{1}$ Namrata Sharma, ${ }^{1}$ \\ Vishal Jhanji $i^{2,3}$
}

1Dr Rajendra Prasad Centre for Ophthalmic Sciences, All India Institute of Medical Sciences, New Delhi, India ${ }^{2}$ Centre for Eye Research Australia, University of Melbourne, Melbourne, Victoria, Australia ${ }^{3}$ Department of Ophthalmology and Visual Sciences, The Chinese University of Hong Kong, Hong Kong, Hong Kong

Correspondence to Professor Rasik B Vajpayee, Dr Rajendra Prasad Centre for Ophthalmic Sciences, All India Institute of Medical Sciences, 474, RP Centre for Ophthalmic Sciences, New Delhi 110029 , India; rasikv@unimelb.edu.au

Received 14 February 2013 Revised 19 March 2013 Accepted 24 March 2013 Published Online First 25 April 2013

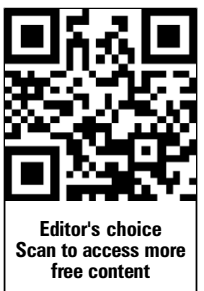

To cite: Vajpayee RB Maharana PK, Kaweri L, et al. Br J Ophthalmol 2013;97:834-836.

\section{ABSTRACT}

Aims To describe a new technique of corneal stab incision with intracameral air injection for management of patients with acute corneal hydrops.

Methods Five patients with acute corneal hydrops with large Descemet's membrane (DM) detachment and multiple stromal clefts underwent the procedure. The technique entailed anterior segment optical coherence tomography guided intrastromal fluid drainage through multiple corneal stromal venting incisions along with anterior chamber air tamponade. The time taken for the DM to reattach, resolution of corneal oedema and the best-corrected visual acuity (BCVA) were assessed postoperatively.

Results Five patients (age range, 10-25 years) with large DM detachment underwent the procedure. The presenting visual acuity varied from hand motions close to face to $1 / 60$. No intraoperative complications were encountered. The DM attached on first postoperative day in four out of five cases. The corneal oedema resolved over 2-3 weeks in all cases. Repeat air injection was not required in any of the cases. All patients had a final $B C V A$ of $\geq 3 / 60$ with two of them achieving a BCVA of $\geq 6 / 24$ at three months postoperatively.

Conclusions The technique of intrastromal drainage of fluid combined with air tamponade can be effectively used as a treatment modality for the management of severe cases of acute corneal hydrops.

\section{INTRODUCTION}

Acute corneal hydrops is a significant complication of non-inflammatory ectatic disorders like keratoconus (KC), pellucid marginal degeneration, Terriens marginal degeneration and keratoglobus. ${ }^{12} \mathrm{~A}$ tear in the Descemet's membrane (DM) with subsequent seepage of aqueous humour in the corneal stroma is the common denominator in all cases. If not treated, resolution usually takes a long time and occurs by endothelial sliding over a period of $2-4$ months. ${ }^{1-3}$ The persistent oedema can cause complications such as corneal neovascularisation, infection and corneal perforation. ${ }^{12}$

Resolution of hydrops can be expedited by intracameral injection of air/isoexpansile gases. ${ }^{1}{ }^{4}$ However, in some cases, presence of large DM detachment or stromal clefts delays the resolution of corneal hydrops. We herein report a technique of anterior segment optical coherence tomography (ASOCT; Visante-OCT; Carl Zeiss Meditec; California, USA) guided intrastromal drainage of fluid combined with intracameral air injection in cases with acute corneal hydrops.

\section{MATERIALS AND METHODS}

Five consecutive patients with acute corneal hydrops with large DM detachment and multiple intrastromal clefts were recruited between January 2012 and September 2012. The study was approved by an institutional review board and followed the tenets of the Declaration of Helsinki. Informed consent was obtained from the patients or the parents of the patients, whenever applicable. The extent of corneal oedema, and if visible, the presence of DM tear and stromal cleft were noted on slit-lamp examination. Corneal oedema was graded according to the grading system proposed by Miyata $e t a l^{5}$ Preoperative uncorrected visual acuity, best- corrected visual acuity (BCVA) and duration of symptoms were recorded. ASOCT was performed preoperatively in all cases. The scans were carefully analysed for the presence of stromal clefts. The highest point of DM detachment relative to the visual axis on the ASOCT scan was noted. A diagrammatic representation of the stromal clefts indicating their position was made on the case files for intraoperative use.

\section{Surgical technique}

Topical or general anaesthesia was used for the surgeries according to the age of the patient. Preoperatively, pupillary constriction was achieved using topical $2 \%$ pilocarpine nitrate at $15-20 \mathrm{~min}$ intervals $1 \mathrm{~h}$ prior to surgery to avoid any intraoperative injury to the lens. After cleaning the eye and periocular area with $5 \%$ povidone iodine, an anterior chamber (AC) paracentesis was performed at 10 o' clock limbus and after expressing out some aqueous humour, the AC was filled with air using a Rycroft cannula mounted on a $5 \mathrm{ml}$ syringe. Subsequently, three to four stab wounds with $45^{\circ}$ angulations in relation to the corneal plane were made in the cornea using anterior segment OCT pictures as a guiding tool. The venting incisions were made in and around the areas of stromal clefts using a 20-gauge microvitreoretinal (MVR) blade (Alcon, Fortsworth, Texas, USA). MVR blade entry was stopped as soon as the egress of fluid was noted through the venting incision. The egressed fluid was dried with a merocel sponge (Madhu instruments, New Delhi, India). After waiting for $10 \mathrm{~min}$, the air was removed partially leaving 
behind an $8 \mathrm{~mm}$ of air bubble in the AC. The paracentesis wound was hydrated using balanced salt solution. Gentamicin $20 \mathrm{mg}$ in $0.5 \mathrm{ml}$ was injected subconjunctivally at the end of the procedure. Postoperatively, topical antibiotics, topical corticosteroids, topical cycloplegics, topical antiglaucoma, and systemic non-steroidal anti-inflammatory drugs were administered.

The status of DM detachment, corneal oedema, intraocular pressure (IOP) and BCVA was recorded on postoperative day 1, week 1, month 1 and, 3 months. ASOCT was performed during all follow-up visits. Resolution of hydrops was defined as the complete disappearance of epithelial and stromal oedema on slit-lamp biomicroscopy and presence of a compact scar with or without vascularisation.

\section{RESULTS}

A total of five patients were included ( 3 men, 2 women; age range 10-25 years). Four patients had $\mathrm{KC}$ and one patient had keratoglobus. Three cases with $\mathrm{KC}$ also had coexistent vernal keratoconjuctivitis. The time duration between the occurrence of hydrops and surgical intervention was between 5 and 12 days. The presenting visual acuity varied from hand motions close to face to $1 / 60$. All procedures were performed successfully using the surgical technique described above. No intraoperative complications were encountered. Postoperatively, the DM was attached on day one in four eyes and on day three in one eye (case 3). The delayed re-attachment in case 3 was possibly related to a very thick cornea preoperatively (table 1 ). The corneal oedema resolved in 2 weeks in 2 eyes and 3 weeks in 3 eyes. No postoperative IOP spike was observed in any of the cases. None of the cases required a repeat air injection (figure 1).

Rapid decrease in corneal oedema with improvement in BCVA was noted in all the cases. The mean corneal thickness decreased from $2936 \pm 1221$ microns preoperatively to $532 \pm 33$ microns at the end of 3 weeks after surgery. All patients had a final BCVA of $\geq 3 / 60$ with two of them achieving a BCVA of $\geq 6 / 24$ at the end of 3 months postoperatively (table 1 ).

\section{DISCUSSION}

Acute corneal hydrops occurs in approximately $2.4-3 \% \%^{12}$ of eyes with $\mathrm{KC}$ and without any intervention it normally resolves over a period of 2-4 months. ${ }^{1} 2$ The various management options to shorten the resolution time include patching, bandage contact lens (BCL) application with topical hypertonic sodium chloride, ${ }^{1} 2$ intracameral injection of air/isoexpansile gas, ${ }^{5-7}$ compressive sutures with gas injection, ${ }^{8}$ amniotic membrane transplantation with cauterisation, ${ }^{9}$ and cyanoacrylate tissue adhesive with BCL. ${ }^{10}$ The average time for resolution of corneal hydrops with these techniques ranges from 2 weeks to 4 months. ${ }^{1}$ In severe cases with multiple stromal clefts and large

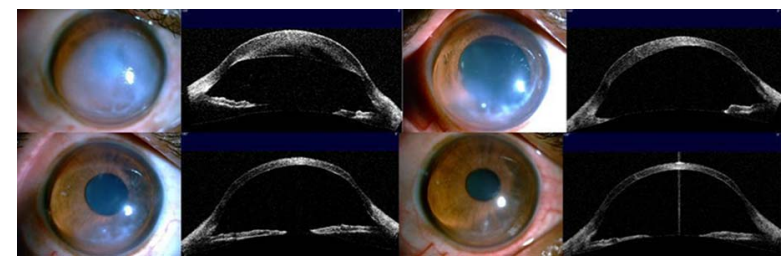

Figure 1 Clinical and anterior segment optical coherence tomography photographs of a representative case of keratoconus with corneal hydrops and intrastromal cleft. Clockwise from top left: at the time of presentation, 1 week after intervention (top right), 1 month after intervention (bottom left) and 3 months after intervention (bottom right). This figure is only reproduced in colour in the online version.

areas of DM detachment, which is often the case in our set up, these conventional procedures are often not effective. Prolonged duration of corneal oedema may be complicated by fistula formation with aqueous leakage, ${ }^{11}$ corneal perforation, ${ }^{1}{ }_{2} \quad 11$ corneal neovascularisation compromising the survival of subsequent grafts ${ }^{12}$ and infection. ${ }^{13}$ Additionally, intracameral injection of air, perfluoropropane (C3F8) and sulfur hexafluoride (SF6) are associated with potential complications like elevation of IOP and pupillary block. ${ }^{15-7}$ Intrastromal migration of air has also been reported. ${ }^{3}$ Decompression sutures carry the risk of suture-related complications, and a second procedure for suture removal is required..$^{8}$ Keratoplasty in an inflamed eye carries a higher risk of rejection. ${ }^{14}$

The basic problem underlying delayed resolution in corneal hydrops is the accumulation of excessive fluid within the intrastromal spaces or clefts. ${ }^{1} 415$ Even if the DM gets reattached with the conventional procedures, it requires a considerable amount of time for this fluid to disappear. A prolonged duration of corneal oedema and inflammation can invite aggressive neovasularisation and its consequent sequale. ${ }^{112}$ Time required for resolution of corneal oedema therefore depends directly on the time required for the detached DM to reattach followed by the absorption of aqueous present within the intrastromal fluid clefts. In our cases, the resolution time of hydrops ranged between 2-3 weeks, which is considerably shorter as compared to studies that used simple air injection as the treatment modality. This was made possible by drainage of fluid from intrastromal clefts using venting incisions. Our technique allows the egress of the intrastromal fluid mechanically, which ensures early resolution of the corneal oedema rather than waiting for the endothelial cells to deturgesce the oedematous cornea. In addition, we believe that iatrogenic stromal scarring induced by venting incisions may result in stronger DM attachment eliminating the need for repeated air/gas injection.

Corneal venting incisions have been successfully used to drain the fluid collecting in the interface area of operated cases of

Table 1 Patient characteristics and outcomes after anterior segment optical coherence tomography guided intrastromal fluid drainage and air tamponade

\begin{tabular}{|c|c|c|c|c|c|c|c|c|}
\hline Case & Diagnosis & $\begin{array}{l}\text { Initial } \\
\text { BCVA }\end{array}$ & $\begin{array}{l}\text { Duration of } \\
\text { symptoms } \\
\text { (days) }\end{array}$ & $\begin{array}{l}\text { Preoperative corneal } \\
\text { thickness (microns) }\end{array}$ & $\begin{array}{l}\text { Descemet's } \\
\text { Reattachment (day) }\end{array}$ & $\begin{array}{l}\text { Time for resolution of } \\
\text { corneal oedema } \\
\text { (weeks) }\end{array}$ & $\begin{array}{l}\text { Postoperative corneal } \\
\text { thickness (microns) } \\
\text { ( } 3 \text { weeks) }\end{array}$ & $\begin{array}{l}\text { Final BCVA } \\
\text { (3 months) }\end{array}$ \\
\hline 1 & KG & $\mathrm{HM}$ & 5 & 1990 & 1 & 2 & 520 & $6 / 18$ \\
\hline 2 & $\mathrm{KC}$ & HM & 10 & 2350 & 3 & 2 & 530 & $3 / 60$ \\
\hline 3 & KC & $\mathrm{PL}+$ & 7 & 4980 & 1 & 3 & 590 & $3 / 60$ \\
\hline 4 & $\mathrm{KC}$ & HM & 12 & 3140 & 1 & 2 & 510 & $6 / 24$ \\
\hline 5 & KC & HM & 6 & 2220 & 1 & 2 & 510 & $4 / 60$ \\
\hline
\end{tabular}

BCVA, best corrected visual acuity; HM, hand motions close to face; KC, keratoconus; KG, keratoglobus; PL, perception of light. 
Descemet's stripping endothelial keratoplasty. ${ }^{16}$ The same principle was applied by us to reattach the DM and hasten the resolution of corneal oedema by creating venting incisions to drain out the fluid from intrastromal clefts. A complete AC air fill for $10 \mathrm{~min}$ gives enough time to drain the intrastromal fluid through the venting incisions and ensures DM reattachment on a permanent basis. Subsequent decompression of air is performed later to prevent pupillary block in the postoperative period. We noted a change in corneal thickness over a period of 3 weeks in all our cases. A potential difficulty during creation of venting incisions is the suboptimal visibility due to extensive corneal oedema. However, we found preoperative localisation of the stromal cleft with ASOCT to be very useful in such cases.

To conclude, our technique of intrastromal drainage of fluid combined with air tamponade is a safe and effective treatment modality for the management of severe cases of acute corneal hydrops.

Contributors RBV: Conceptualized the idea, performed surgeries and final preparation of manuscript. PM: Helped in preoperative workup and follow-up of cases and drafted the manuscript. NS: Helped in recruitment and follow-up of patients and revision of manuscript. LK: helped in preoperative workup and follow-up of cases. VJ: Writing of the manuscript, critical revision of the manuscript, final approval of the manuscript, logistical support.

Competing interests None.

Ethics approval Ethics Committee of All India Institute of medical Sciences.

Provenance and peer review Not commissioned; externally peer reviewed.

\section{REFERENCES}

1 Sharma N, Maharana PK, Jhanji V, et al. Management of acute corneal hydrops in ectatic corneal disorders. Curr Opin Ophthalmol 2012;23:317-23.
2 Grewal S, Laibson P, Cohen E, et al. Acute hydrops in the corneal ectasia: associated factors and outcomes. Trans Am Ophthalmol Soc 1999;97:187-98.

3 Sharma N, Mannan R, Titiyal JS. Nonresolution of acute hydrops because of intrastromal migration of perfluoropropane gas. Cornea 2010;29:944-6.

4 Sharma N, Mannan R, Jhanji V, et al. Ultrasound biomicroscopy-guided assessment of acute corneal hydrops. Ophthalmology 2011;118:2166-71.

5 Miyata K, Tsuji H, Tanabe T, et al. Intracameral air injection for acute hydrops in keratoconus. Am J Ophthalmol 2002;133:750-2.

6 Panda A, Aggarwal A, Madhavi P, et al. Management of acute corneal hydrops secondary to keratoconus with intracameral injection of sulfur hexafluoride (SF6). Cornea 2007:26:1067-9.

7 Basu S, Vaddavalli PK, Ramappa M, et al. Intracameral perfluoropropane gas in the treatment of acute corneal hydrops. Ophthalmology 2011;118:934-9.

8 Rajaraman R, Singh S, Raghavan A, et al. Efficacy and safety of intracameral perfluoropropane ( $\mathrm{C} 3 \mathrm{~F} 8$ ) tamponade and compression sutures for the management of acute corneal hydrops. Cornea 2009;28:317-20.

9 Wylegala E, Tarnawska D. Amniotic membrane transplantation with cauterization for keratoconus complicated by persistent hydrops in mentally retarded patients. Ophthalmology 2006;113:561-4.

10 Sii F, Lee GA, Gole GA. Perforated corneal hydrops treated with sulfur hexafluoride (SF6) gas and tissue adhesive. Cornea 2005;24:503-4.

11 Aldave AJ, Mabon M, Hollander DA, et al. Spontaneous corneal hydrops and perforation in keratoconus and pellucid marginal degeneration. Cornea 2003;22:169-74

12 Rowson NJ, Dart JK, Buckley RJ. Corneal neovascularisation in acute hydrops. Eye 1992;6:404-6.

13 Donnefeld ED, Schrier A, Perry HD, et al. Infectious keratitis with corneal perforation associated with corneal hydrops and contact lens wear in keratoconus. $\mathrm{Br} J$ Ophthalmol 1996:80:409-12.

14 Cobo LM, Coster DJ, Rice NS, et al. Prognosis and management of corneal transplantation for herpetic keratitis. Arch Ophthalmol 1980;98:1755-9.

15 Feder RS, Wilhelmus KR, Vold SD, et al. Intrastromal clefts in Keratoconus patients with hydrops. Am J Ophthalmol 1998;126:9-16.

16 Srinivasan S, Rootman DS. Slit-lamp technique of draining interface fluid following Descemet's stripping endothelial Keratoplasty. Br J Ophthalmol 2007;91:1202-5. 